\title{
CHEMOTHERAPY OF VENEREAL DISEASES ITS USES AND ABUSES
}

\author{
BY \\ S. M. LAIRD
}

Ipswich

For centuries the man of medicine practised the relief of symptoms, his power to influence the processes of disease being strictly limited ; the art of medicine was largely confined to providing circumstances favourable for the vis medicatrix naturae to function to the best advantage. When, however, Pasteur, Lister, and Koch turned the spot-light of science on the bacterial battlefield, knowledge of the biology of infections began to grow. The biochemical search for specific remedies and the birth of chemotherapy during the last half-century were inevitable developments. Salvarsan and the later arsphenamine preparations, the bismuth compounds, the sulphonamides, and the antibiotics are all potent weapons in the fight against the bacterial enemies of man; their use involves relatively new and fundamental principles. As in modern warfare, the discoveries of the " backroom boys" must undergo carefully studied field trials, and the lessons learnt from such trials must then be assimilated by those who will play their part in the front line. Otherwise the new weapons may not be used to full advantage and disaster may overtake those that abuse them.

\section{Application of Chemotherapy}

The mode of action of chemotherapeutic agents on bacteria is not yet known in detail ; there is evidence, however, that the drugs may interfere with the surface structure of the bacterial cell and with the enzyme actions necessary for its normal metabolism. We are all familiar with the morphological and tintorial changes which the gonococcus may manifest under the influence of sulphonamides, and the electron microscope has shown aberrant forms and division of the Nichols strain of $T$. pallidum in contact with penicillin. The important result of these intrinsic effects is that in practice the chemotherapeutic agent may be bacteriostatic, or bactericidal, or both. The aim of chemotherapy is, therefore, to provide an effective tissue level of the drug at the site of infection and to maintain this level until the infective agent has been eliminated. When the organism is sensitive and the drug is bactericidal this period may be very short, but where the action of the drug is merely bacteriostatic it must be administered over longer periods so that the natural defence mechanisms of the host may have time to overcome the infection. Three important factors are thus concerned : the efficacy of the drug; the virulence of the organism; and the natural defensive powers of the patient.

Efficacy of the Drug.-This depends on the sensitivity of the organism and on the tissue level which can be established and maintained at the site of infection. The tissue level is usually lower than the blood level of the drug but if the site of infection is vascular enough these levels will approximate closely to one another. The persistence of the tissue concentration at a higher level than that of the blood concentration after administration of the drug has ceased, is an important practical observation. The blood level achieved depends on the frequency of administration and on the rate of absorption and excretion of the drug used. In the case of crystalline penicillin $\mathrm{G}$ in aqueous solution, both absorption and excretion are rapid, and 3hourly administration of 40,000 units is required to maintain an effective therapeutic level; even an individual dose of 500,000 units must be repeated after only 8 hours. Attempts to delay excretion are open to objection, and more practical progress has followed the use of absorption-delaying vehicles. Of these preparations, procaine penicillin in oil with 2 per cent. aluminium monostearate $(300,000 \mathrm{u} / \mathrm{ml}$.$) , alone or reinforced with crystal-$ line penicillin $G(100,000 \mathrm{u} / \mathrm{ml}$.), gives adequate results when injected every 24 or even 48 hours in 2-ml. doses. More recently, aqueous procaine penicillin preparations have become available, 
and these are reported to be more convenient to administer and, on in vitro testing, to be as effective as the oily procaine penicillin. The peak level achieved by these delayed-absorption preparations is much lower than that of crystalline penicillin in aqueous solution, and, in acute infection or where the organism is relatively insensitive, there is a danger that effective use may be sacrificed to the convenience of administration. The more rapid absorption in ambulant patients as compared with the bed-patient must also be remembered.

Penicillin can be given by mouth, but its oral use is wasteful, less certain than parenteral administration, and more open to abuse. Instillation into the conjunctival sac and the use of penicillin ointment for blepharitis and other eye conditions are justifiable exceptions to the rule that penicillin should only be given by injection. The widespread prescription of penicillin ointment for local application to surface lesions, often not infective in origin, is an outstanding abuse of chemotherapy, which daily causes danger and acute distress, from sensitization, to hundreds of patients.

Virulence of Infecting Organism.-The strength of the infection and its sensitivity to chemotherapeutic drugs are fundamentally important. Drug-sensitivity, or conversely drug resistance, is usually relative rather than absolute. Most of us have had first hand experience of the development of drugresistance in connexion with the sulphonamide treatment of gonorrhoea. This phenomenon has probably resulted from the steady elimination of sensitive strains from the human population, and from the encouragement of the gonococcus to develop drug-resistance by irregular and inadequate dosage arising from attempts at self-treatment or prophylaxis and, if the truth is told, from uninformed medical prescribing. There is little sign so far, of the gonococcus or T. pallidum becoming resistant to penicillin, but the demonstration of strains of staphylococci and other bacteria developing such resistance should serve as a warning of this dangerous possibility.

Natural Defence.-The third fundamental in chemotherapy comprises the natural defensive power of the patient. This factor is vital where the drug action is wholly or largely bacteriostatic. I believe that sometimes insufficient attention is given to it in the assessment of the results obtained with a particular treatment schedule. We have all seen the case of early syphilis cured with minimum treatment while other patients fail to respond to much more adequate therapy. I believe that such instances arise from variation between individuals in their natural powers of recovery, and moreover that any treatment schedule for any infection will have its failures or relapses.

\section{General Principles}

The fundamentals considered above provide certain principles which must be observed faithfully in the use of chemotherapy.

(1) The infective agent must be identified bacteriologically and its sensitivity to chemotherapeutic agents determined, either by direct laboratory investigation or in the light of past clinical experience.

(2) Having considered which chemotherapeutic agent to employ, the best method of administration must be chosen with reference to route, individual and total dosage, frequency, and duration. The biology and location of the infection, the resultant tissue pathology, and possible toxic reactions to treatment must all receive attention.

(3) The patient's general condition must receive all necessary care to promote the highest possible level of well-being and encourage his natural powers of resistance ; such attention may need to embrace psychological and social factors.

(4) Where the infection may be contagious, as with the venereal diseases, epidemiological investigation of intimate contacts and the prevention of re-infection are often necessary.

(5) As chemotherapy is not invariably successful, the patient must receive adequate observation to establish cure or to detect relapse or failure without delay.

\section{Practical Application}

Observance of these principles constitutes the proper use of chemotherapy ; their non-observance, means its abuse. Their practical application to certain particular venereal diseases may be briefly outlined as follows :

Gonorrhoea.-Diagnosis must never be based solely on clinical findings ; smears and, certainly in the female, cultures are essential for pre-treatment diagnosis and post-treatment follow-up. One injection of $1 \mathrm{ml}$. (400,000 units) procaine penicillin reinforced with crystalline penicillin $G$ is effective in uncomplicated infection in the male. For the female I prefer one dose of 800,000 units. When complications are present, more than one such injection may be required combined with local treatment and I believe this to be particularly true of salpingitis.

The higher initial blood level achieved by the reinforcement of procaine penicillin with the crystalline penicillin $G$ is of especial value in gonorrhoea. In all cases I think it valuable to 
combine the penicillin with "Sulphatriad", $4 \mathrm{~g}$. daily for five days, partly because the latter is often more effective than penicillin against secondary organisms, and partly because the combination of two or more chemotherapeutic agents is thought to reduce greatly the possibility of development of drug-resistant strains.

In non-gonococcal urethritis in the male I use streptomycin only after penicillin and Sulphatriad have failed, giving $1 \mathrm{~g}$. daily for not more than three days. In my experience, gonococcal rheumatism and Reiter's disease still require artificial fever and meticulous management of the genito-urinary focus ; here chemotherapy, including chloromycetin and aureomycin, has proved disappointing.

Syphilis.-My early experience of the treatment of primary and secondary syphilis with sixty injections amorphous penicillin (total 2.4 mega units in $7 \frac{1}{2}$ days) with a relapse rate of some 12 to 14 per cent. quickly led me to combine penicillin with arsenic and bismuth. From 1945 to 1948 my treatment of early syphilis comprised 100 injections penicillin (4 mega units) combined with one course of ten weekly injections of neoarsphenamine and bismuth. This schedule gave excellent results in 97 per cent. of patients, the failures (less than 3 per cent.) all arising in examples of late secondary syphilis. From May, 1948, such cases of late secondary syphilis have been given two courses of metal chemotherapy with improved results. Towards the end of 1949 an increasingly reassuring follow-up of my own cases and mounting experience reported from the U.S.A. led me to abandon the use of the arsenicals in the belief that 600,000 units procaine penicillin can achieve as much as one injection of neoarsphenamine with less danger of toxic reaction. I still prefer to admit to hospital all cases of early syphilis, especially where pregnancy co-exists. To such patients I give 100 injections crystalline penicillin $G$ in aqueous solution (total 4 mega units), and at the same time I begin a course of ten weekly injections $0 \cdot 2 \mathrm{~g}$. bismuth oxychloride. After discharge from hospital the patient is given ten weekly injections of 600,000 units procaine penicillin so that the whole schedule takes about 12 weeks to complete. If the patient is unable to come into hospital he is given 600,000 units of procaine penicillin daily for 15 days and then ten weekly injections. In pregnant women treatment should be maintained up to the time of confinement.

The value of penicillin in neurosyphilis and optic atrophy seems very considerable, and I have also thought it of benefit in cardiovascular syphilis. Preliminary preparation with bismuth is essential to reduce Herxheimer reactions and prevent a therapeutic paradox and I can think of no case of late syphilis of such urgency that this important preliminary to penicillin is precluded.

I began my remarks with a reference to the vis medicatrix naturae and its all important role in the patient's recovery. It is still vital but now we can assist it greatly by the intelligent use of chemotherapy. These drugs have eased the patient's burden immensely but their proper usage in assisting his recovery has placed an added responsibility on his doctor which was unknown to the practitioner of earlier days. Let us abuse neither the responsibility nor the drugs. 